\title{
Outcomes of Medicare Outpatient Total Knee Arthroplasty at an Ambulatory Surgery Center
}

\author{
Daniel Lo ${ }^{1 *}$, Amber McDermott ${ }^{1}$, William Durkin ${ }^{1}$ and Dean Marshall ${ }^{2}$ \\ ${ }^{1}$ Department of Orthopaedic Surgery, Aultman Hospital, USA \\ ${ }^{2}$ Department of Orthopaedic Surgery, Spectrum Orthopaedics, Ohio Specialty Surgical Suites, \\ USA
}

*Corresponding author: Daniel Lo, Department of Orthopaedic Surgery, Aultman Hospital, 2600 6th SW, Canton, OH, USA, Tel: 1 (330) 452-9911; Email: Daniel.Lo@aultman.com

\section{Research Article \\ Volume 5 Issue 1}

Received Date: February 18, 2021

Published Date: March 03, 2021

DOI: $10.23880 /$ jobd-16000204

\section{Abstract}

Background: Total knee arthroplasty (TKA) was removed from the "inpatient only" list for Medicare Beneficiaries in 2018. As a result, outpatient TKA's have been performed at ambulatory surgery centers (ASC) more frequently. This study aims to evaluate outcomes of medicare patients who underwent outpatient TKA at an ASC.

Methods: We conducted a retrospective cohort review of medicare patients who underwent TKA at an ASC between January 1st, 2020 and June 30th, 2020 performed by six orthopedic surgeons.

Results: Thirty-six patients were identified who underwent primary TKA. There was a mean age of 72.4 and body mass index of 30.9. The mean preoperative range of motion was -6.7 degrees of extension and 114.8 degrees of flexion, two and six week post operative extension of -3.8 degrees and flexion of 104 degrees and -2.7 degrees and 114.6 degrees respectively. Preoperative physical and mental patient reported outcomes measurement and information system scores were 43.2 and 53.4 respectively and 49.4 and 53.1 post operatively. Preoperative patient reported outcomes measure with the knee injury and osteoarthritis outcome score was 48.4 and 72.8 post operatively. There were two patients (5.5\%) with complications of arthrofibrosis that required manipulation under anesthesia. Total recovery time and time within the ASC were on average 200 minutes and 398.6 minutes respectively.

Conclusion: Without adverse events within the first six months, this study suggests that outpatient TKA can be safely performed in medicare patients at an ASC

Keywords: Total Knee Arthroplasty; Complications; Outpatient; Ambulatory Surgery Center; Medicare

\section{Introduction}

Total knee arthroplasty (TKA) is one of the most common elective surgeries performed in the United States today with more than one million procedures performed every year [1]. This number will only increase with predictions of an increase of volume near $143 \%$ by 2050 with the aging population [2]. Total knee arthroplasty was recently taken off of the "inpatient only" list January 1, 2018 by the Centers of Medicare and Medicaid Services [3]. Since then, there has been a considerable amount of confusion causing a disruption to the hospital, surgeons, staff and patients $[4,5]$. Furthermore, 


\section{Journal of Orthopedics \& Bone Disorders}

beginning in January of 2020, TKA has also been added to the covered surgical procedure list at Ambulatory Surgery Centers (ASC). It has been well documented that outpatient TKA have comparable immediate post-operative outcomes but these results are largely based on outpatient hospital procedures [6-9]. While these studies are useful, there is little to no published research providing results of immediate post-operative outcomes in medicare patients who undergo TKA at an ambulatory surgery center. The safety of major surgeries at an ambulatory surgery center continues to be of question throughout the community. The objective of this research is to evaluate a cohort of medicare patients who underwent total knee arthroplasty at an ambulatory surgery center and report on postoperative outcomes up to 6 months of follow up.

\section{Materials and Methods}

We conducted a retrospective cohort study by manually reviewing and extracting electronic medical records on medicare patients who underwent total knee arthroplasty. Institutional review board approval was obtained. A total of thirty-six patients underwent TKA who met operative indications between January 1st, 2020 to June $30^{\text {th }}, 2020$. These surgeries were performed by a total of six surgeons at a single ambulatory surgical center. These were the first 36 medicare patients that were approved to have their TKA performed at the ASC. Inclusion criteria were medicare patients who underwent a primary TKA during the first six months of the year 2020. All patients who underwent TKA at the ambulatory surgery center were reviewed and those who did not have Medicare insurance were excluded from the study. Patients underwent evaluation by their primary care providers, other established medical providers, and were met by anesthesia prior to their surgery date. The surgery center has been performing outpatient TKA's from 2015 through 2019 and have performed 579 successful TKA's in privately insured patients. An established protocol is in place to optimize patient selection. All patients who met preoperative requirements to have surgery performed at an ASC were allowed to proceed.

A midline, medial parapatellar approach was performed using surgeon preferred instrumentation and implants. All patients were managed with a standardized surgeon specific rehabilitation protocol both pre and post operatively. Preoperative data including patient demographics, laterality, ASA class, preoperative range of motion, 2 week and 6 week post-operative range of motion, complications within the first 6 months, readmission rates, total and recovery time in the ambulatory surgical center. At the preoperative visit, all patients received both a physical and mental Patient Reported Outcome Measurement and Information System (PROMIS) and Patient reported outcomes via Knee Injury and Osteoarthritis Outcome Score (KOOS). Both systems are reported to be validated scoring systems in patients undergoing total knee arthroplasty $[10,11]$. Post operatively; all patients received online PROMIS and KOOS scores that the patient completed at 2 weeks, 6 weeks, 3 months, and 6 months. Patients underwent surgeon specific physical therapy protocols.

\section{Results}

There were thirty-six patients with end stage osteoarthritis who failed nonoperative treatment. The mean age was 72.4 years old with an average BMI of $30.9+/-5 \mathrm{~kg} /$ $\mathrm{m}^{2}$. There were 15 males (42\%) and 21 females (58\%) with 19 being left knees (53\%) and 17 being right (47\%). There were 15 patients with an ASA score of II and 21 patients with an ASA score of III. The demographics of the patients are displayed in Table 1. The preoperative range of motion of the knee was $-6.6+/-6.6$ degrees of extension and 114.8 + - 12.2 degrees of flexion. The preoperative physical and mental PROMIS scores were $43.2+/-7$ and $53.4+/-7.1$ and the preoperative KOOS scores were $48.4+/-9.4$. The range of motion at 2 and 6 weeks were $-2.7+/-3.3$ degrees of extension and $104+/-11.6$ degrees of flexion and $-2.7+/-3.3$ degrees of extension $114.6+/-11.1$ degrees of flexion respectively. The preoperative and postoperative comparisons can be found in Table 2. The total and recovery time in the ambulatory surgical center were $398.6+/-47.8$ minutes and $200.3+/$ 42.1 minutes respectively. Time spent at the ASC can be found in Table 3. Two patients (5.5\%) had complications of arthrofibrosis; both of which were treated with manipulation under anesthesia. Complications are found in Table 4. No patients throughout the study were noted to have any major complications including return to the operating room, wound related infection, thromboembolic events, sepsis, and death. There were zero incidents of intraoperative complications, blood transfusions, admission to the hospital, adverse events within the ASC, VTE, or readmissions for postoperative pain.

\begin{tabular}{|c|c|c|}
\hline \multicolumn{3}{|c|}{ Demographics } \\
\hline Age in years & $72.4+/-4.8$ & \\
\hline Gender & Male: 15 & Female: 21 \\
\hline Body Mass Index & $30.9+/-5$ & \\
\hline Side & Left: 19 & Right: 17 \\
\hline ASA Class & II: 15 & III: 21 \\
\hline \multicolumn{3}{|c|}{ Data are expressed as a mean of standard deviation +/- } \\
\hline \multicolumn{3}{|c|}{ ASA, American Society of Anesthesiologist physical } \\
Classification system \\
\hline
\end{tabular}

Table 1: The demographics of the patients. 
Journal of Orthopedics \& Bone Disorders

\begin{tabular}{|c|c|}
\hline Characteristics & Scores \\
\hline Preoperative ROM & $\begin{array}{c}6.6+/-6.6-114.8+/-12.2 \\
\text { degrees }\end{array}$ \\
\hline $\begin{array}{l}\text { Postoperative ROM at } 2 \\
\text { weeks }\end{array}$ & $\begin{array}{l}2.7+/-3.3-104+11.6 \\
\text { degrees }\end{array}$ \\
\hline $\begin{array}{c}\text { Postoperative ROM at } 6 \\
\text { weeks }\end{array}$ & $\begin{array}{c}2.7+/-3.3-114.6+/-11.1 \\
\text { degrees }\end{array}$ \\
\hline $\begin{array}{l}\text { Preoperative PROMIS } \\
\text { Physical }\end{array}$ & $43.2+/-7$ \\
\hline $\begin{array}{l}\text { Postoperative PROMIS } \\
\text { Physical }\end{array}$ & $49.4+/-6.7$ \\
\hline $\begin{array}{l}\text { Preoperative PROMIS } \\
\text { Mental }\end{array}$ & $53.4+/-7.1$ \\
\hline $\begin{array}{l}\text { Postoperative PROMIS } \\
\text { Mental }\end{array}$ & $53.1+/-6.4$ \\
\hline Preoperative PROM-KOOS & $48.4+/-9.4$ \\
\hline $\begin{array}{l}\text { Postoperative PROM- } \\
\text { KOOS }\end{array}$ & $72.8+/-14.8$ \\
\hline \multicolumn{2}{|c|}{$\begin{array}{l}\text { PROM-KOOS = Patient reported outcomes measure with } \\
\text { The Knee Injury and Osteoarthritis Outcome Score }\end{array}$} \\
\hline \multicolumn{2}{|c|}{$\begin{array}{l}\text { PROMIS = Patient Reported Outcomes Measurement and } \\
\text { Information System that consists of both physical and } \\
\text { mental health }\end{array}$} \\
\hline \multicolumn{2}{|c|}{$\begin{aligned} \text { ROM = } & \text { Range of Motion, Format: extension with standard } \\
& \text { deviation to flexion with standard deviation }\end{aligned}$} \\
\hline Data are expressed & 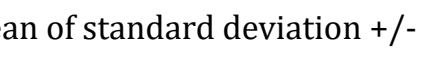 \\
\hline
\end{tabular}

Table 2: The preoperative and postoperative comparisons.

\begin{tabular}{|c|c|}
\hline & Time \\
\hline Total Time in minutes & $398.6+/-47.8$ \\
\hline Recovery Time in minutes* & $200.3+/-42.1$ \\
\hline \multicolumn{2}{|c|}{ Data are expressed as a mean of standard deviation $+/-$} \\
\hline *Starts with patient time out of operating room to time of \\
discharge \\
\hline Data are expressed as a mean of standard deviation $+/-$ \\
\hline
\end{tabular}

Table 3: Time spent at the ASC.

\begin{tabular}{|c|c|c|}
\hline Number & $\begin{array}{c}\text { Complications } \\
\text { at 180 days }\end{array}$ & Treatment \\
\hline $2(5.5 \%)$ & Arthrofibrosis & $\begin{array}{c}\text { Manipulation under } \\
\text { anesthesia }\end{array}$ \\
\hline
\end{tabular}

Table 4: Complications.

\section{Discussion}

There have been many studies that demonstrate outpatient total knee arthroplasty is a safe effective procedure that does not increase readmission rates [12-14]. The safety of total joint arthroplasty performed at ASC has been questioned with a considerable amount of confusion on financial implications as performing these surgeries in this setting is still in its infancy $[15,16]$. There have been studies that demonstrate safety of outpatient TKA in medicare patients but these patients had the safety net of having the ability to stay overnight at the hospital with inpatient and/ or observation capabilities [17]. However, limited studies have been published examining the outcomes of medicare patients who undergo outpatient TKA specifically at an ambulatory surgery center. The primary purpose of this study was to demonstrate the safety of outpatient TKA in medicare patients at an ambulatory surgery center.

The first aim was specifically to characterize the occurrence rate of post-operative complications that occurred within the six month postoperative period in our medicare patients who underwent outpatient total knee arthroplasty at the ambulatory surgery center. An analysis of postoperative complications and treatment are listed in Table 4. There were no documented complications of readmission, wound related infection, thromboembolic events, sepsis and death.

Of the thirty-six patients, there were a total of two $(5.5 \%)$ that had a postoperative complication, both of which suffered from arthrofibrosis. It is important for patients to maintain functional range of motion of their knee. Preoperatively, our patients had an average range of motion of $-6.6+/-6.6$ degree of extension and $114.8+/-12.2$ degrees of flexion seen in Table 2. It has been documented that elderly patient's minimal functional range of motion is around 90-110 degrees of flexion [18,19]. Arthrofibrosis can be a debilitating complication after undergoing a TKA with a reported incidence from 1-13\% [20-23]. Both were treated successfully with manipulation under anesthesia without need for further surgery. These findings are reassuring and supportive of the safety of TKA in medicare patients at an ambulatory surgery center with comparable arthrofibrosis rates in the historical literature.

The current study has limitations. The sample size of our patients was relatively small so it may not be representative of a larger cohort. This is in part due to the time frame of our study as it took place during the COVID-19 pandemic. This caused a large number of cases to be cancelled at both our institution and nationally [24]. The pandemic also limited in-person followup after six weeks. Patients who 


\section{Journal of Orthopedics \& Bone Disorders}

were subjectively doing "well" were given the option to follow up via telemedicine. This could potentially lead to underreported complications.

\section{Conclusion}

Outpatient total knee arthroplasty has been an increasingly popular procedure in medicare patients with increased utilization of ambulatory surgery centers. This study suggests that outpatient TKA can be safely performed in medicare patients at an ambulatory surgery center. Complication rates are comparable to the established literature without an increase in any major complications or adverse events within the first six months of followup.

\section{References}

1. Steiner C, Andrews R, Barrett M, Weiss A (2012) HCUP Projections: Mobility/Orthopedic Procedures 2003 to 2012. 2012. HCUP Projections Report \# 2012-03. US. Agency for Healthcare Research and Quality.

2. Inacio MCS, Paxton EW, Graves SE, Namba RS, Nemes S (2017) Projected Increase in Total Knee Arthroplasty in the United States-an Alternative Projection Model. Osteoarthritis and Cartilage 25(11): 1797-1803.

3. Fact Sheet CY (2020) Medicare Hospital Outpatient Prospective Payment System and Ambulatory Surgical Center Payment System Final Rule (CMS-1717-FC). CMS.

4. Yates Adolph J, Joshua M Kerr, Mark I Froimson, Craig J Della Valle, James I Huddleston (2018) The Unintended Impact of the Removal of Total Knee Arthroplasty From the Center for Medicare and Medicaid Services Inpatient-Only List. The Journal of Arthroplasty 33(12): 3602-3606.

5. Krueger Chad A, Joshua M Kerr, Michael P Bolognesi, Maxwell Courtney P, James I Huddleston (2020) The Removal of Total Hip and Total Knee Arthroplasty From the Inpatient-Only List Increases the Administrative Burden of Surgeons and Continues to Cause Confusion. The Journal of Arthroplasty 35(10): 2772-2778.

6. Bovonratwet Patawut, Nathaniel T Ondeck, Stephen J Nelson, Jonathan J Cui, Matthew L Webb, et al. (2017) Comparison of Outpatient vs Inpatient Total Knee Arthroplasty: An ACS-NSQIP Analysis. The Journal of Arthroplasty 32(6): 1773-1778.

7. Aynardi Michael, Zachary Post, Alvin Ong, Fabio Orozco, Dean C Sukin (2014) Outpatient Surgery as a Means of Cost Reduction in Total Hip Arthroplasty: A Case-Control Study." HSS Journal ® 10(3): 252-255.
8. Berger Richard, Sheila Sanders, Eileen D'Ambrogio, Kate Buchheit, Carl Deirmengian, et al. (2010) Minimally Invasive Quadriceps-Sparing TKA: Results of a Comprehensive Pathway for Outpatient TKA. The Journal of Knee Surgery 19(02): 145-148.

9. Parcells Bertrand W, Dean Giacobbe, David Macknet, Amy Smith, Mark Schottenfeld, et al. (2016) Total Joint Arthroplasty in a Stand-Alone Ambulatory Surgical Center: Short-Term Outcomes. Orthopedics 39(4): 223228.

10. Lyman Stephen, Yuo-Yu Lee, Patricia D Franklin, Wenjun Li, Michael B Cross, et al. (2016) Validation of the KOOS, JR: A Short-Form Knee Arthroplasty Outcomes Survey. Clinical Orthopaedics and Related Research ${ }^{\circledR}$ 474(6): 1461-1471.

11. Khalil Lafi S, Brian Darrith, Sreten Franovic, Jason J Davis, Robb M Weir, et al. (2020) Patient-Reported Outcomes Measurement Information System (PROMIS) Global Health Short Forms Demonstrate Responsiveness in Patients Undergoing Knee Arthroplasty. The Journal of Arthroplasty 35(6): 1540-1544.

12. Kelmer Grayson C, Justin J Turcotte, Paul J King (2020) Same-Day vs One-Day Discharge: Rates and Reasons for Emergency Department Return After Hospital-Based Total Joint Arthroplasty. The Journal of Arthroplasty 36(3):879-884.

13. Courtney P Maxwell, Mark I Froimson, R Michael Meneghini, Gwo-Chin Lee, Craig J Della Valle (2018) Can Total Knee Arthroplasty Be Performed Safely as an Outpatient in the Medicare Population? The Journal of Arthroplasty 33(7S): S28-S31.

14. Shah, Ritesh R, Nancy E Cipparrone, Alexander C Gordon, David J Raab, James R Bresch (2018) Is It Safe? Outpatient Total Joint Arthroplasty with Discharge to Home at a Freestanding Ambulatory Surgical Center. Arthroplasty Today 4(4): 484-487.

15. Chambers Monique, James I Huddleston, Mohamad J Halawi (2020) Total Knee Arthroplasty in Ambulatory Surgery Centers: The New Reality!. Arthroplasty Today 6(2): 146-148.

16. Rodríguez-Merchán, Carlos E (2020) Outpatient Total Knee Arthroplasty: Is It Worth Considering? EFORT Open Reviews 5(3): 172-179.

17. Springer Bryan D, Susan M Odum, David N Vegari, Jeffrey G Mokris, Walter B Beaver (2017) Impact of Inpatient Versus Outpatient Total Joint Arthroplasty on 30-Day Hospital Readmission Rates and Unplanned Episodes of 


\section{Journal of Orthopedics \& Bone Disorders}

Care. Orthopedic Clinics of North America 48(1): 15-23.

18. Rowe PJ, Myles CM, Walker C, Nutton R (2000) Knee Joint Kinematics in Gait and Other Functional Activities Measured Using Flexible Electrogoniometry: How Much Knee Motion Is Sufficient for Normal Daily Life? Gait Posture 12(2): 143-155.

19. McClelland Jodie A, Julian A Feller, Hylton B Menz, Kate E Webster (2017) Patients with Total Knee Arthroplasty Do Not Use All of Their Available Range of Knee Flexion during Functional Activities. Clinical Biomechanics 43: 74-78.

20. Donaldson JR, Tudor F, Gollish J (2016) Revision Surgery for the Stiff Total Knee Arthroplasty. The Bone \& Joint Journal 98-B(5): 622-627.

21. Manrique Jorge, Paul E Di Cesare (2014) Stiffness after
Total Knee Arthroplasty. Journal of Knee Surgery 28(02): 119-126.

22. Yercan Huseyin S, Tahir S Sugun, Christophe Bussiere, Tarik Ait Si Selmi, Andrew Davies, et al. (2006) Stiffness after Total Knee Arthroplasty: Prevalence, Management and Outcomes. The Knee 13(2): 111-117.

23. Desai Aravind S, Anand Karmegam, Asterios Dramis, Tim N Board, Videsh Raut (2014) Manipulation for Stiffness Following Total Knee Arthroplasty: When and How Often to Do It? European Journal of Orthopaedic Surgery \& Traumatology 24(7): 1291-1295.

24. Bedard Nicholas A, Jacob M Elkins, Timothy S Brown (2020) Effect of COVID-19 on Hip and Knee Arthroplasty Surgical Volume in the United States. The Journal of Arthroplasty 35(7): S45-S48. 